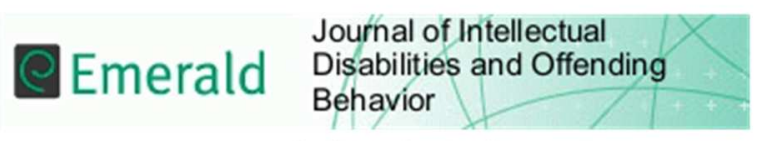

\title{
The validity of two diagnostic systems for personality disorder in people with intellectual disabilities: a short report
}

\begin{tabular}{|r|l|}
\hline Journal: & Journal of Intellectual Disabilities and Offending Behavior \\
\hline Manuscript ID & JIDOB-04-2015-0004.R1 \\
\hline Manuscript Type: & Research Paper \\
\hline Keywords: & $\begin{array}{l}\text { Personality disorder, clinical assessment, structured assessment, Learning } \\
\text { disability, offenders, intellectual disability }\end{array}$ \\
\hline \multicolumn{2}{|c}{} \\
\hline
\end{tabular}

\section{SCHOLARONE ${ }^{m}$ \\ Manuscripts}


The validity of two diagnostic systems for personality disorder in people with intellectual disabilities: a short report

\section{Abstract}

Background -- Over the last 10 years there has been greater interest in the diagnosis of personality disorder (PD) in people with intellectual disabilities (ID). One important characteristic of a diagnostic system is that it should have validity as a contribution to utility. PD has been found to have a predictive relationship with violence and the present study reviews two methods for the diagnosis of PD in offenders with ID in order to evaluate the utility of the diagnoses.

Method --212 offenders with ID were recruited from three settings -- maximum-security, medium/low security and community services. Three research assistants were trained over a period of two weeks in order to increase reliability for the extraction of information from the case notes and the diagnosis of PD. Diagnoses of PD in the case files were compared with a structured system of diagnosis based on DSM IV traits.

Results - There were significant differences between the two systems with a significantly higher frequency of PD diagnosis in the community forensic setting in the structured assessment system. There was no relationship between the case files diagnosis of PD and future violence but there was a significant predictive relationship between the structured diagnosis of $\mathrm{PD}$ and future violence with an $\mathrm{AUC}=.62$.

Conclusions -- Only the structured assessment of PD had utility for the prediction of violence. Reasons for the differences between the systems are discussed and suggestions made on how a diagnosis of PD can be structured for the busy clinician. 
Diagnosis is one of the cornerstones of clinical practice. A diagnosis should be as concise as possible and a technical summary that conveys information about the cause, nature and symptoms of the disorder. It should provide a "roadmap" that gives an indication of any follow-up tests required, treatment or management that might be indicated, future manifestations and prognosis of the disorder. For many disorders these are ideals that are only achieved occasionally and for psychiatric disorders, they are rarely seen completely. However, for many conditions they are reasonable goals. For example, a diagnosis of intellectual disability provides summary information, or at least broad predictions about intellectual capacity, adaptive behaviour, likely parameters across the lifespan, and likely responses to educational and occupational opportunities. Therefore a diagnosis carries information about how the disorder may have developed in the past, current manifestations and prediction of future implications.

The diagnosis of personality disorder (PD) in people with intellectual and developmental disabilities (IDD) has been particularly problematic because of lack of reliability and validity. Studies have found huge differences in prevalence in what were similar populations. For example, Eaton \& Menolascino (1982) reported a prevalence rate of $27 \%$ for PD in a community based sample of 115 people with IDD and Ballinger \& Reid (1987) reported a prevalence rate of PD in 22\% of 100 individuals with mild or moderate IDD. On the other hand, Goldberg, Gitta \& Puddephatt (1995) found abnormal personality traits in 57\% of individuals in an institutional sample and $91 \%$ of individuals in a community sample. Flynn, Matthews \& Hollins (2002) studied a hospital inpatient sample and reported that $92 \%$ were diagnosed with PD. A turning point in research on PD and IDD came with Alexander \& Cooray (2003) review of studies. They noted that there was a lack of reliable diagnostic instruments, the use of different diagnostic systems (ICD-10 and DSM III \& IV ), a confusion of definition and personality theory and difficulty in distinguishing PD from other problems integral to IDD such as communication problems, sensory disorders and developmental delay. They concluded that "the variation in the co-occurrence of personality disorder in learning disability, with prevalence ranging from less than $1 \%$ to $91 \%$ in a community setting and $22 \%$ to $92 \%$ in hospital settings, is very great and too large to be explained by real differences" (p s28). They recommended 
tighter diagnostic criteria and greater use of behavioural observation and informant information in addition to interviewing which is the principle method for diagnosis.

Lindsay et al. (2006) employed the recommendations made by Alexander \& Cooray (2003) in a study of 164 males with IDD in three forensic settings-high secure, medium/low secure and community forensic services. They employed four independently rated measures of PD: a DSM IV criteria checklist completed firstly from file review, secondly by a clinician and thirdly from nurse observations and finally the Structured Assessment of Personality (SAP; Mann et al 1981) completed by care staff. A consensus rating was derived from the four assessments and a total prevalence of PD in this forensic sample was $39.5 \%$. They reported that the ratings had high levels of interater reliability. As would be expected in a forensic population, antisocial personality disorder was the largest category at $22 \%$ of cases and rates of PD across the other categories were between 1 and $3 \%$. Prevalence in this forensic sample was similar to that found by other research groups in mainstream forensic samples without IDD (e.g. Blackburn et al., 2006).

. In clinical practice, in most cases a PD diagnosis is established on interview only. This is understandable since clinicians are busy and seldom have time for gathering information from multiple sources. Paris (2013), an acknowledged expert on PD diagnosis and treatment, stated "few practitioners have followed the relatively simple algorithms of DSM III or DSM IV as directed (because) they only have a short time to make any diagnosis" (p170) Given that several authors (Alexander \& Cooray 2003; Reid et al., 2004) have recommended a more extensive procedure such as that used by Lindsay et al. (2006) for the diagnosis of PD in people with IDD it remains to be seen which method is more consistent with the requirements and functions of diagnosis set out in the first paragraph. The present study employed an extensive database on 164 offenders with IDD in which there were two systems recorded for the diagnosis of PD: a clinical diagnosis taken from the case files which was generally established through interview and based on ICD 10 criteria and a diagnosis based on four sources of information. 
As indicated in the first paragraph, a diagnostic system should enable prediction of certain related factors and since PD has been strongly associated with violence in offender populations the prediction of violence (Monahan et al 2001; Harris, Rice \& Quinsey 1993) has been focussed on in this study. The relationship between PD and offending was reinforced by the review on PD and IDD presented by Raynor et al (2015) when they found that the presence of PD in someone with IDD is associated with more dangerous offending. The present study is a comparison of the predictive value of these two methods in relation to future violence. .

Method

Settings and participants. The study assessed 212 adult males across three forensic IDD services. Of these, 73 participants were drawn from a high security site $(\mathrm{H})$ which is the national centre for high secure IDD patients. Patients were generally referred from prisons, secure hospitals, and courts. The service accepted patients deemed to present a grave and immediate danger to the public. All patients were considered for escorted ground access within the perimeter; only a small number had individual escort, and a few had unescorted status. All education, therapy, vocational, and leisure facilities are provided within the secure perimeter by staff employed or contracted to the organisation to meet the specific needs of the resident patient population. An additional 70 participants were drawn from a medium/low secure hospital (M/L) which provides in patient forensic services on a local, regional, and national basis. Patients were generally referred via health authorities, the courts, and prisons. Medium secure services function independently within the hospital and all day services including occupation, education, leisure, and sport are provided within the unit. Low secure services include designated units and rehabilitation apartments. The final 69 participants were drawn from a community forensic IDD service (C) which had been in existence for 13 years. There was a small 10-bedded open unit and a larger number of day places associated with the service. Most patients are assessed and treated while maintaining their community placement.

There was no difference between the groups on IQ $(\mathrm{H}=66.7 ; \mathrm{M} / \mathrm{L}=66.6 ; \mathrm{C}=64.7 ; \mathrm{F}=1.07$, $\mathrm{p}=0.35$ ). Patients from $\mathrm{C}$ were significantly younger than those in the other two settings (average 
$34 \mathrm{v} 39$ years; $\mathrm{F}=3.75, \mathrm{p}=0.025$; medium effect size). There were no significant differences between the groups in terms of the percentage who had committed any sexual offence. Patients from C had committed fewer serious sexual assaults but this was not statistically significant $(H=15.1 ; M / L=12.9$; $\mathrm{C}=5.8 ; \mathrm{p}=0.19$ ). A significantly higher percentage of participants in $\mathrm{H}$ had committed violent offences $(\mathrm{p}=0.005$; large effect size) and a significantly lower percentage of participants in $\mathrm{M} / \mathrm{L}(\mathrm{p}=0.01$; large effect size) were diagnosed with major mental illness (psychotic disorders or major mood disorders). Unfortunately, due to participant refusal, insufficient information in casenotes, inability to contact carers, and difficulty in contacting psychiatrists/psychologists who had left the services, only 164 completed sets of data for structured assessment of PD were available $(H, n=53 ; M / L, n=41 ; C, n=69)$. Applications for ethical approval were made and granted locally at each site.

Assessments.

Clinical diagnosis. - Clinical diagnosis, generally made by the consultant psychiatrist on the basis of interview, was extracted from the case files. Three research assistants conducted the study, one in each setting. Each research assistant had at least degree-level psychology training and extensive further training was provided for the purposes of this project on DSM-IV personality diagnosis. One full week's training was given on measures and a second week was given over to assessing inter-rater reliability between research assistants. This involved data collection and scoring on three cases across a range of measures. A file review was conducted as part of the study. Reliability was calculated for 120 cases. The percentage agreement for the recording of personality disorder in the case file was $97 \%$. Agreement was calculated by dividing the number of agreements by the number of agreements plus disagreements expressed as a percentage.

Structured diagnosis - DSM-IV personality disorder consensus diagnosis (D’Silva \& Hogue, 2002) was used in addition to the Standardised Assessment of Personality (SAP; Pilgrim \& Mann, 1990). This diagnostic system comprises four sets of separate diagnoses from which a final diagnosis of DSM-IV criteria personality disorder was obtained. All final diagnoses were made by the research assistant, independent of raters. Each symptom from each of the 10 PD diagnoses in DSM-IV was presented in a standardised fashion to each assessor. One assessment was completed from a file review, a second with a clinician familiar with the participant (psychiatrist/psychologist), a third with 
nursing or care staff who knew the individual (observer rating), and a fourth was completed via a SAP interview with direct care staff. The final judgement of the presence or absence of the 93 individual traits (corresponding to DSM-IV) comprising the diagnostic system was determined through a process of structured clinical judgement and was made independently of staff who knew the participant. Final diagnoses were made on the 11 types of PD (including PD not otherwise specified) as identified by DSM-IV guidelines.

Inter-rater reliability. One measure of inter-rater reliability was calculated from two independent sources of diagnosis. The decisions made on the basis of information contained in the file and decisions made by independent clinicians were compared to assess the reliability of rater judgements on the presence or absence of PD. Cohen's kappa (k) was calculated on each PD category. However, low base rates observed for most PDs in this study generally resulted in low kappa values despite high agreement between raters. If one considers the agreed absence of PD to be a significant result, then percentage agreement is an acceptable measure. Since we noted in the introduction that some studies have reported an extremely high prevalence of PD in this client group, then agreed absence is indeed an important finding. Therefore, both kappas and percentage agreement are reported. Percentage agreement between clinician ratings and ratings based on file information was calculated using the formula total number of agreements divided by the total number of agreements plus disagreements, expressed as a percentage. Reliability for individual PD classifications ranged from $76 \%$ to $98 \%$ with overall reliability $87 \%$. Kappas ranged from .32 to .77 with oveall Kappa $=$ .56 (medium effect size). If the participant fulfilled criteria for a specific personality disorder on three of the four measures, the research assistant then coded that participant as having the relevant personality disorder diagnosis.

Incident recording - Violent incident data were extracted from the official systems for recording incidents at each of the three sites. It is important to note that diagnosis of PD was independent of recording of violent incidents in the present study. The records contained a description of the incident, the individuals involved and any resulting injuries. From this information, raters manually scored the data into yes or no for whether the individuals engaged in violent behaviour.

\section{Results.}


Table 1 around here.

In the results to follow $\mathrm{H}$ represents the maximum secure participants, $\mathrm{M} / \mathrm{L}$ the medium/low secure participants and $\mathrm{C}$ the community participants. Table 1 shows the percentages with actual numbers in parenthesis of PD diagnosed under each system (structured diagnostic system V case files) for each subgroup and for the total cohort. It should be remembered that in the DSM categories there was missing data (number stated above). There was a higher rate of PD diagnosis in structured assessment system (41.9\%) when compared to the case file (25.1\%). This is due to the lower frequency of diagnosis in the case file, especially in the community setting (C) and, to a lesser extent, in the medium/low secure setting (M/L). Only one individual in $\mathrm{C}$ was diagnosed with PD. Using chi square, the differences between the two systems of diagnosis is highly significant for the total cohort. When comparing the separate settings, $\mathrm{C}$ showed a significant difference using Fisher's exact probability test because of the low numbers in one cell.

As mentioned in the introduction, PD has been found to have significant association with violence in that several authors have found PD to be a moderate predictor of violence in criminal populations. We therefore used receiver operator characteristics (ROC) to calculate the extent to which a diagnosis of PD and each system predicted future of violent incidents. Violent incident data were as coded as occurring for that individual (yes) or not occurring (no). Of the 212 participants, 157 were scored as yes, 48 as no, and there were missing data for 7 participants.

For the total cohorts, ROC Area Under the Curve (AUC) was $\mathrm{AUC}=.47(\mathrm{p}=0.45)$ for the case file diagnosis; and $\mathrm{AUC}=.62(\mathrm{p}=0.01)$ for the structured DSM IV diagnosis. Therefore, only the structured diagnostic system predicted future violent incidents. The AUCs for both analyses can be seen in figure 1.

Figure 1 around here.

For the separate settings numbers were smaller and so none of the AUCs reached significance but for comparison they are reported. For the Case file system the AUCs for $\mathrm{H}=.46, \mathrm{M} / \mathrm{L}=.36$ and $\mathrm{C}=.52$. For the structured system the AUCs were $\mathrm{H}=.65, \mathrm{M} / \mathrm{L}=.62$ and $\mathrm{C}=.63$. Therefore the results returned from the individual settings were consistent with the overall AUCs.

Discussion 
The present study has been able to compare two systems for the diagnosis of personality disorder using the same cohort of participants. The two systems are firstly, routine diagnosis that has been extracted from the case file and, secondly, a structured assessment using four different sources of data. To recap, there was a significant difference between the two systems. In the community, only one offender with IDD was recorded with a diagnosis of PD in the case files. On the other hand, structured assessment resulted in $41 \%$ of participants in $\mathrm{C}$ being diagnosed with PD. In the maximum security setting there was little difference between the two diagnostic systems in terms of total percentages $(57 \% \mathrm{~V} 55 \%)$. In the medium/low secure setting there was a doubling of prevalence under the structured system.

One possible reason for the difference in the frequency of PD diagnosis between the two systems might be attributed to the settings. In maximum security settings clinicians are very comfortable and familiar with the concept of PD. Indeed, it is essential to have a diagnosis of mental disorder for admission to maximum security hospital and some of these patients are likely to be detained for reasons of personality disorder. Therefore clinicians are required to be very familiar with both legislation and the diagnoses. In community settings this is far less true. In our experience, even clinicians in community forensic IDD services do not consider personality disorder with the same familiarity as those in medium or maximum secure settings. Clinicians in community settings are much more likely to be familiar with concept of mental illness as part of dual diagnosis with ID and for this reason, PD may be somewhat underdiagnosed. In addition, PD is a very pejorative label which conjures up images of violence and self harm. Although many studies have shown that PD is both manageable and treatable (Paris, 2013; Lindsay et al., 2013), the concept invokes images of severe dysfunction. Clinicians in community settings are often seeking placements for their patients with voluntary services and community services and may be reluctant to use a pejorative diagnosis of PD because it will act as a disincentive to any receiving service. These effects may have contributed to underdiagnosis of $\mathrm{PD}$ in the case files for the community forensic service.

The most important question, however, is which system better fulfils the requirements of diagnosis. One crucial function of diagnosis is that it should broadly predict the course and consequences of the disorder. One important consequence in the literature is that PD is associated 
with violence in men. Only the structured system predicted future violence with a small to medium effect size. In fact PD is included in the main violence risk assessments (the VRAG and the HCR20).

It is included because studies have found a relationship between PD and violence with small effect sizes (Harris et al., 1993, 2015; Monahan et al., 2001). Therefore it is crucial that a diagnosis of PD has validity and the system which had greatest validity in the present study is undoubtedly the structured diagnosis of PD. The diagnoses extracted from the case files did not predict incidents at a rate better than chance.

While it is the case that only the structured system had predictive validity, the system used in the current study is far too unwieldy for routine clinical practice. Four independent assessments were conducted on each personality trait included in each PD diagnoses for DSM IV. The busy clinician would simply not have time for this. We would therefore recommend that the clinician should consider recommendations made by several reviewers (Alexander \& Cooray 2003; Reid et al 2004; Lindsay \& Alexander 2015) to include staff observations and clinician assessments. We would also suggest the new system under DSM-5 (Amerian Psychiatic Association 2013) which at first appears complicated but is in fact quite straightforward in its application. The system employs a dimensional approach to personality dysfunction (from normal personality to extreme impairment) but does retain six personality disorders -- antisocial, avoidant, borderline, narcissistic, obsessive compulsive and schizotypal. Although there are several stages to the diagnostic system, only the first four are absolutely necessary. The first step is to familiarise oneself with the assessment of Severity of Personality Function which is described in DSM-5 and ranges from normal functioning, through mild, moderate and severe, to extreme impairment of personality. The second is an appraisal of which maladaptive personality traits are present, thirdly, if personality functioning is maladaptive, making a decision on whether he/she conforms to any of 6 retained PD diagnoses, and finally deciding on the dysfunctional traits that comprise a possible diagnosis of PD trait specified when the person does not fit any of the 6 categories of PD. The traits specified conform to the 5 factor model of personality that has emerged from the extensive research on personality (Blackburn 2000; Costa \& McCrae 1995). Therefore, in essence, the clinician should perform a structured assessment based on the traits that constitute the six retained PD diagnoses using the framework for the Severity of Personality 
Functioning assessment. The way in which this diagnostic structure can be adapted for people with IDD has been described by Lindsay and Alexander (2015).

References.

Alexander, R., \& Cooray, S. (2003). Diagnosis of personality disorders in learning disability. British Journal of Psychiatry, 182 (Suppl. 44), S28-S31.

American Psychiatric Association (2013) Diagnostic and statistical manual of mental disorders. Fifth edition (DSM 5). Arlington, VA. American Psychiatric Association.

Ballinger, B. R., \& Reid, A. H. (1987). A standardised assessment of personality in mental handicap. British Journal of Psychiatry, 150, 108-109.

Blackburn, R. (2000). Classification and assessment of personality disorders in mentally disordered offenders: A psychological perspective. Criminal Behaviour \& Mental Health, 10, (Special Suppl.), S8-S32.

Blackburn, R., Logan, C., Renwick, S.J.D., \& Donnelly, J.P. (2005). Higher order dimensions of personality disorder: Hierarchical and relationships with the five factor model, the interpersonal circle and psychopathy. Journal of PersonalityDisorders, 19, 597-623.

Costa, P.T. Jr., \& McCrae, R.R. (1995). Domains and facets: Hierarchical personality assessment using the revised NEO Personality Inventory. Journal of Personality Assessment, 64(1), 2150. 
D’Silva, K., \& Hogue, T. (2002). Directions for the classification of DSM-IV personality disorder diagnoses. Unpublished manuscript, available from the authors.

Eaton, I.F., \& Menolascino, F.J. (1982). Psychiatric disorders in the mentally retarded: Types problems and challenges. American Journal of Psychiatry, 139, 1297-1303.

Flynn, A., Mathews, H., \& Hollins, S. (2002). Validity of the diagnosis of personality disorder in adults with learning disability and severe behaviour problems. British Journal of Psychiatry, $180,543-546$.

Goldberg, B., Gitta, M. Z., \& Puddephatt, A. (1995). Personality and trait disturbance in an adult mental retardation population: Significance for psychiatric management. Journal of Intellectual Disability Research, 39, 284-294.

Harris, G.T., Rice, M.E., \& Quinsey, V.L. (1993). Violent recidivism of mentally disordered offenders: The development of a statistical prediction instrument. Criminal Justice \& Behaviour, 20, 315-335.

Harris, G.T., Rice, M.E., Quinsey, V.L \& Cormier, C.A. (2015). Violent offenders: Appraising and managing risk. Washington DC: American Psychological Association.

Hogue, T.E., Steptoe, L., Taylor, J.L., Lindsay, W.R., Mooney, P. \& Pinkney, L. (2006). A comparison of offenders with intellectual disability across three levels of security. Criminal Behaviour and Mental Health, 16, 13-28.

Landis, J. R., \& Koch, G. G. (1977). The measurement of observer agreement for categorical data. Biometrics, 33, 159-174.

Lindsay, W.R. \& Alexander, R. (2015) Personality Disorders. In C. Hemmings \& N. Bouras (Eds.) Psychiatric and Behavioural Disorders in Intellectual and Developmental Disabilities, $3^{\text {rd }}$ Edition. Cambridge, UK. Cambridge Univ. Press

Lindsay W. R., Hogue T., Taylor J.T., Steptoe L., Mooney P., Johnston S., O’Brien G. \& Smith A. H.W. (2006) Two studies on the prevalence and validity of personalitydisorder in three forensic learning disability samples. Journal of Forensic Psychiatry and Psychology 17, $485-506$. 
Lindsay W.R., Steptoe, L., Wallace L., Haut F. \& Brewster, E. (2013) An evaluation and 20 year follow up of recidivism in a community intellectual disability service. Criminal Behaviour and Mental Health, 23, 138-49,

Monahan, J., Steadman, H., Silver, E., Appelbaum, P., Robbins, T., Mulvey, E., Roth, L., Grisso, T., \& Banks, S. (2001). Re-thinking risk assessment: The MacArthur study of mental disorder and violence. New York: Oxford University Press.

Mann, A. H., Jenkins, R., Cutting, J. H., \& Cowan, P. J. (1981). The development and use of a standardised assessment of abnormal personality. Psychological Medicine, 11, 839-847.

Paris, J. (2013a) The intelligent clinician's guide to the DSM 5. Oxford University press: Oxford

Pilgrim, J., \& Mann, A. (1990). Use of ICD-10 version of the standardised assessment of personality to determine the prevalence of personality disorder in psychiatric in-patients. Psychological Medicine, 20, 985-992.

Rayner, K., Wood, H., Beail, N. \& Kaur Nagra , M. (2015). Intellectual disability, personality disorder and offending: a systematic review. Advances in Mental Health and Intellectual Disabilities, 9, $50-61$

Reid, A. H., Lindsay, W. R., Law, J., \& Sturmey, P. (2004). The relationship of offending behaviour and personality disorder in people with developmental disabilities. In W. R. Lindsay, J. L. Taylor, \& P. Sturmey (Eds.), Offenders with developmental disabilities (pp. 289-303). Chichester, UK: Wiley \& Sons. 
Table 1. Chi Square Test for Personality Disorder from Case File and DSM-IV Assessment across settings, including Percentage Prevalence (Actual Numbers in parenthesis), chi square value and significance levels.

\begin{tabular}{llllll} 
Setting & $\begin{array}{l}\text { \%PD in case } \\
\text { files (ICD 10) }\end{array}$ & $\begin{array}{l}\text { \%PD } \\
\text { DSM IV }\end{array}$ & $\chi^{2}$ & $d f$ & Sig. \\
\hline Total (212) & $25.3(48)$ & $41.9(67)$ & 11.32 & 1 & .001 \\
$\mathrm{H}$ & $57.1(40)$ & $54.9(28)$ & 0.02 & 1 & .88 \\
$\mathrm{M} / \mathrm{L}$ & $14(7)$ & $28.0(11)$ & 2.17 & 1 & .16 \\
$\mathrm{C}$ & $1.5(1)$ & $40.6(28)$ & FE & 1 & .000 \\
\hline
\end{tabular}

Study Settings: $\mathrm{H}=$ high security; $\mathrm{M} / \mathrm{L}=$ medium and low security; $\mathrm{C}=\mathrm{Community}$ 
Figure 1. AUCs for the prediction of violent incidents for both the case file and DSM IV systems of PD diagnosis.

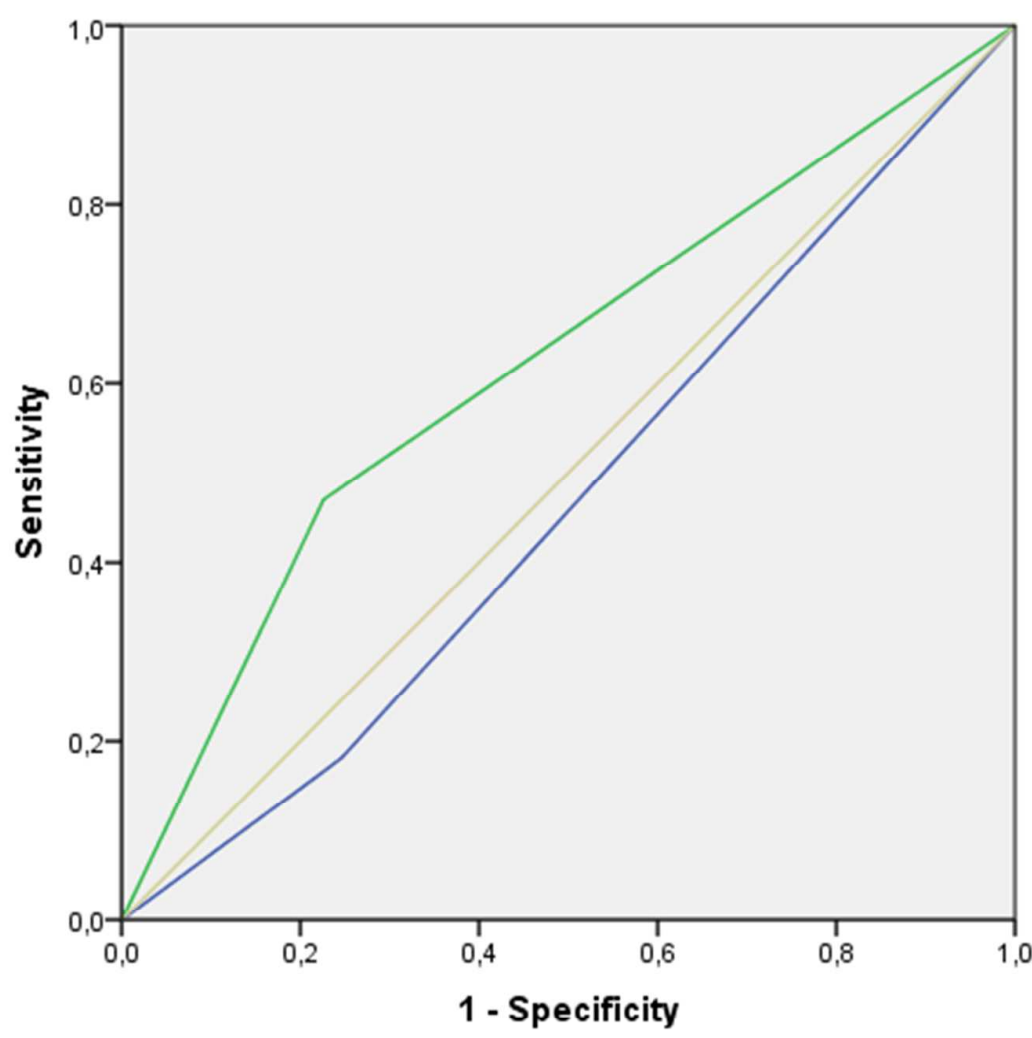

Source of the Curve

- Case File (ICD-10 assessment)

- DSM-IV assessment 